\title{
Capillarity-assisted fabrication of nanostructures using a less permeable mold for nanotribological applications
}

\author{
Kahp Y. Suh ${ }^{\text {a) }}$ and Hoon Eui Jeong \\ School of Mechanical and Aerospace Engineering and the Institute of Advanced Machinery and Design, \\ Seoul National University, Seoul 151-742, Korea
}

Deok-Ho Kim ${ }^{\text {b) }}$

Microsystem Research Center, Korea Institute of Science and Technology, Seoul, 136-791, Korea

\section{R. Arvind Singh and Eui-Sung Yoon}

Tribology Research Center, Korea Institute of Science and Technology, Seoul, 136-791, Korea

(Received 22 February 2006; accepted 10 May 2006; published online 3 August 2006)

\begin{abstract}
A simple kinetic model is presented to describe the capillary rise of a thin polymer film into a less permeable polyurethane acrylate mold. In this model, capillarity is explained by the competition between capillary and hydrodynamic forces in the course of pattern formation. For a less permeable mold, it was found that the capillary rise increases linearly with time. In addition, the contribution of viscosity and film thickness disappears such that the kinetics is solely governed by the permeation kinetics and capillary force. The present model would be useful to describe the evolution of molded nanostructures when a less permeable mold material other than polydimethylsiloxane is used for the patterning. Moreover, nanostructures with different tip shapes (rounded or dimpled) were observed depending on the fabrication temperature. The structures were tested for potential nanotribological applications such as reduction in adhesive and friction forces. (C) 2006 American Institute of Physics. [DOI: $10.1063 / 1.2222071]$
\end{abstract}

\section{INTRODUCTION}

Recently, capillarity has been frequently used to fabricate polymeric micro/nanostructures. When a liquid wets a cavity with lowering of the free energy, the wetting leads to the capillary movement of the liquid. ${ }^{1}$ Typically, the curvature induced Laplace pressure is counterbalanced by gravitational force (vertical capillary rise) or trapped air or defects in the void spaces (lateral capillary flow). The use of capillarity for microfabrication was first introduced in a method called micromolding in capillaries. ${ }^{2}$

Recently, by combining essential features of nanoimprint and capillarity, we developed capillary lithography for fabricating polymeric micro/nanostructures over large areas. ${ }^{3-6}$ In this method, we directly placed a patterned elastomeric mold onto a spin-coated thermoplastic resin on a substrate and then formed a negative replica of the mold by raising the temperature above the resin's glass transition temperature after solvent evaporation (temperature-induced capillarity), ${ }^{3}$ or direct molding prior to solvent evaporation (solvent-induced capillarity). ${ }^{4}$ A potential concern in this technique is that a high aspect-ratio sub-100 nm PDMS mold is nearly impossible to obtain due to the low elastic modulus of polydimethylsiloxane (PDMS) $(\sim 1.8 \mathrm{MPa})$. Several researchers reported that PDMS gives rise to deformation, buckling, or collapse of shallow relief features ${ }^{7,8}$ or high surface tension causes rounding of sharp corners when released from the master. ${ }^{9}$

\footnotetext{
${ }^{\text {a) }}$ Author to whom correspondence should be addressed; electronic mail sky4u@snu.ac.kr

${ }^{b}$ Present address: Department of Biomedical Engineering, Johns Hopkins University, Baltimore, Maryland.
}

To overcome these mechanical shortcomings of PDMS, a number of alternative molds have been presented such as a composite PDMS ${ }^{10}$ a bilayer PDMS, ${ }^{11}$ a photocurable PDMS, ${ }^{12}$ a polyolefin mold, ${ }^{13}$ a PDMS with polymerreinforced sidewalls, ${ }^{14}$ a Teflon mold, ${ }^{15,16}$ and a photocurable polyurethane acrylate mold. ${ }^{17,18}$ Of these, the polyurethane acrylate (PUA) mold turned out to be stiff enough for replicating dense sub-100 nm features. In addition, the mold allows for flexibility that is capable of large area replication and a tunable modulus, enabling from imprinting to microcontact printing (tensile modulus ranges from 19.8 to $320 \mathrm{MPa}) .{ }^{19}$

For PDMS molds, air can easily permeate through the mold since PDMS is presumably the most permeable rubbery polymer to air. ${ }^{20}$ For less permeable materials such as PUA and polyolefin, however, the permeability kinetics should be taken into account to describe the capillary rise of polymer melt over time. In general, capillary rise is hindered by air permeation because the hydrodynamic pressure builds up as the capillary rise progresses. To gain an understanding on the interplay between capillarity and air permeation, we recently derived a capillary kinetics based on the Poiseuille formulation when a liquid rises into an end-capped cavity of the mold. ${ }^{21}$ It was predicted that the rate of capillary rise would increase linearly with time when the gas permeation is extremely small as opposed to a parabolic increase for high air permeation (e.g., for PDMS). This parabolic increase was in good agreement with the capillary rise of a polymer melt into PDMS cavity. ${ }^{6,21}$ Here, we endeavor to test for the validity of the model by fabricating nanostructures with various aspect ratios (up to $\sim 5$ ) using a less permeable PUA mold. In addition, the molded nanostructures can be fabricated with 
different tip shapes (rounded or dimpled), providing potential nanotribological applications such as reduction in adhesive and friction forces. The current capillary system allows for excellent flexibility towards nanotribology without tailoring the shape of mold and specific polymer materials as described below.

\section{EXPERIMENTAL METHOD}

\section{A. Fabrication of PUA mold}

We fabricated PUA molds that were comprised of polyurethane prepolymers, a monomeric modulator, photoinitiator and a radiation curable releasing agent as reported previously. ${ }^{18}$ The original silicon master was prepared by photolithography with the target geometry of protruding $250 \mathrm{~nm}$ pillars with $500 \mathrm{~nm}$ pillar-to-pillar spacing. However, the master was over etched during reactive ion etching, rendering nanopillars of $\sim 150 \mathrm{~nm}$ at base and $\sim 50 \mathrm{~nm}$ at top and a height of $\sim 500 \mathrm{~nm}$. To fabricate a sheet-type mold, the liquid mixture was drop dispensed onto the silicon master pattern and then a flexible, transparent polyethylene terephthalate (PET) film was brought into contact with the liquid mixture. Subsequently, it was exposed to UV ( $\lambda$ $=200-400 \mathrm{~nm}$ ) for a few tens of seconds through the transparent backplane (dose $=100 \mathrm{~mJ} / \mathrm{cm}^{2}$ ). After the UV curing, the mold was peeled off from the master pattern. The resulting PUA mold was a thin sheet with the thickness of $\sim 50 \mu \mathrm{m}$ and had a height of $\sim 480 \mathrm{~nm}$, slightly lower than that of the original master. The tensile modulus of the mold was measured to be $\sim 320 \mathrm{MPa}$. The number of mold that can be made from a single master was limitless as long as the silicon master stayed clean.

\section{B. Fabrication of nanostructures of PMMA}

For patterning, cleaned silicon substrates were spin coated with a $10 \%$ poly(methylmethacrylate) (PMMA) solution $\left(M_{w}=120000 \mathrm{~g} / \mathrm{mol}, T_{g}=105^{\circ} \mathrm{C}\right.$, Aldrich $)$ in toluene. The samples were, in turn, baked in a vacuum oven at $100{ }^{\circ} \mathrm{C}$ for $2 \mathrm{~h}$ to remove any residual solvent in the film. A PUA mold was then placed on the polymer under a slight pressure $\left(\sim 10 \mathrm{~g} / \mathrm{cm}^{2}\right)$ to make conformal contact. To investigate the effects of temperature on aspect ratio and shape of nanopillars, the samples were annealed at different temperatures from 110 to $160{ }^{\circ} \mathrm{C}$ at atmospheric pressure. While maintaining the temperature above $T_{g}$, the sample was left undisturbed for various annealing times from $10 \mathrm{~min}$ to $24 \mathrm{~h}$. The resulting patterns were examined by scanning electron microscopy (SEM, XL30FEG, Philips Electron Co., Netherlands).

\section{Measurements of adhesion and friction properties}

After pattern formation, the static contact angles of water were measured with a contact anglemeter (ELMA, Goniometer Type) to evaluate the hydrophobicity of the surface. Using the sessile-drop method, the measurements were repeated at least five times for each sample. The patterns were also tested for adhesion and friction properties (load $0-80 \mathrm{nN}$, speed $2 \mu \mathrm{m} / \mathrm{s}$, scan size $5 \mu \mathrm{m} \times 5 \mu \mathrm{m}$ ), at nano-

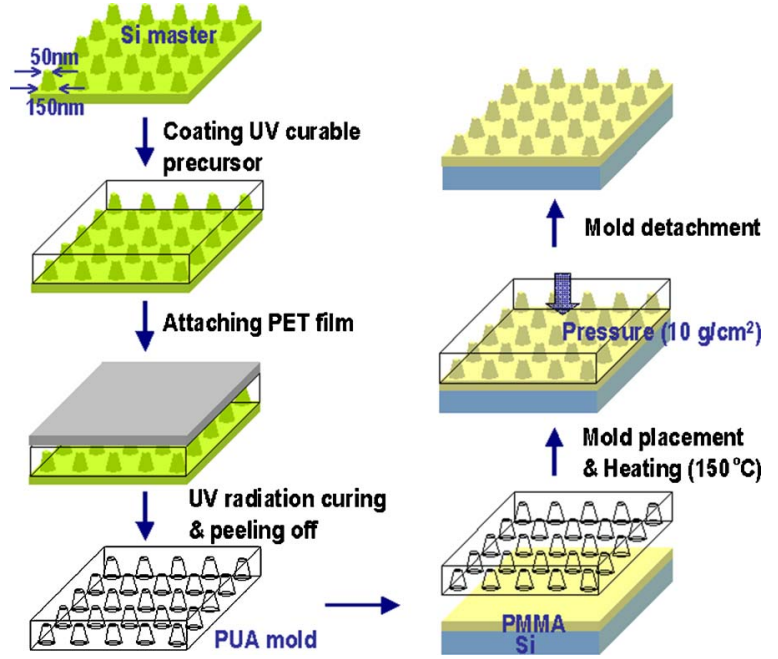

FIG. 1. (Color online) A schematic illustration of the fabrication procedure. The PUA mold used in the experiment is a negative thin sheet (features sticking in). See the experimental procedure for the detailed process.

scale using atomic force microscopy (AFM, Nanoscope IIIa, Digital Instruments). Borosilicate ball (radius $1.25 \mu \mathrm{m}$ ) mounted on cantilever (contact mode type NPS, Digital Instruments) was used as a tip. At microscale, friction was measured using a soda lime ball (radius $0.5 \mathrm{~mm}$, Duke Scientific Corp.) under reciprocating motion (load $3000 \mu \mathrm{N}$, speed $1 \mathrm{~mm} / \mathrm{s}$ ) using a microtribo tester. All experiments were conducted at controlled temperature $\left(24 \pm 1{ }^{\circ} \mathrm{C}\right)$ and humidity $(45 \pm 5 \%)$.

\section{RESULTS AND DISCUSSION}

\section{A. Fabrication of PMMA nanostructures}

A schematic diagram of the capillarity-assisted molding procedure is shown in Fig. 1. The original Si master has cone-shaped protruding cylinders on the surface from excessive reactive ion etching. The protruding cylinders were exactly replicated in the PUA mold with height reduction less than 5\%. A PET film was applied on the uncured PUA layer as a supporting substrate to ease the release of the cured film. The resulting PUA mold is a high-resolution, flexible thin sheet that can make a conformal contact with a smooth solid surface under a slight physical pressure. To yield a uniform pressure distribution, a thin slab of PDMS buffer layer $(\sim 0.5 \mathrm{~cm})$ was placed on top of the PUA mold prior to the application of pressure.

Figure 2 shows SEM images for the evolution of $150 \mathrm{~nm}$ nanopillars with time obtained at $120{ }^{\circ} \mathrm{C}$. Since heat was conducted directed from a hot stage to the sample, the incubation time needed to arrive at the target temperature would be minimal. It can be seen from the figure that the height or aspect ratio was proportional to annealing time and the PMMA film reached the ceiling of the mold at $<\sim 60 \mathrm{~min}$. At initial stage, the rate of capillary rise at the mold wall was faster than that at the center due to the presence of a meniscus as seen from Figs. 2(a)-2(c). After reaching the ceiling of the mold, the PMMA melt reflowed to fill the entire space of 
(a) $\mathrm{t}=5 \mathrm{~min}$

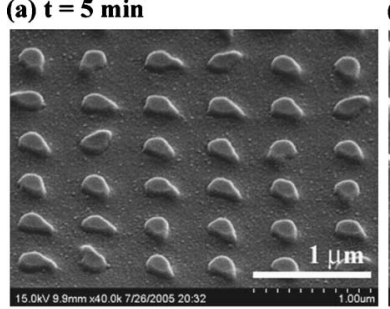

(c) $\mathrm{t}=\mathbf{3 0}$ min

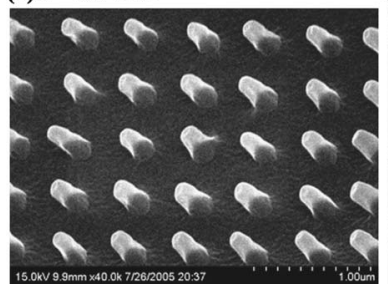

FIG. 2. Tilted SEM images for the evolution of $150 \mathrm{~nm}$ PMMA pillars with time at $120^{\circ} \mathrm{C}$. A meniscus was seen from (a) to (c) while the polymer completely filled the cavity after $60 \mathrm{~min}$.

the void [Fig. 2(d)]. The maximum height was measured to be $\sim 460 \mathrm{~nm}$, in good agreement with the height of the original PUA mold $(\sim 480 \mathrm{~nm})$.

\section{B. Capillary kinetics with less permeable PUA mold (PET support)}

According to a previous report, the capillary kinetics for less permeable mold is given by ${ }^{21}$

$$
z=\frac{2 P e \gamma \cos \theta}{d R} t=(P e / d) \Delta P_{L} t
$$

where $\mathrm{z}$ is the distance of polymer movement in time, $P e$ is the permeability of the mold to air, $\gamma$ is the surface tension of the polymer, $\theta$ is the equilibrium contact angle at the threephase contact line, $d$ is the permeation length of the mold, $R$ is the radius of the pillar, and $\Delta P_{L}$ is the Laplace pressure $(=2 \gamma \cos \theta / R)$. The Laplace pressure was estimated from Young's equation such that the effects of a slanted wall and nonuniformly curved meniscus were not taken into consideration. The actual Laplace pressure would be somewhat smaller. Equation (1) indicates that the capillary rise increases linearly with time for a given permeability and Laplace pressure. The physical interpretation is that air permeates out of the cavity through the mold with Laplace pressure being a driving potential. In addition, the relation suggests that the contribution of viscosity and film thickness disappears such that the kinetics is solely governed by the permeation kinetics and capillary force.

A number of physical properties are needed to evaluate the capillary kinetics. First, the permeability of the mold is mostly governed by the PET film since a PET film of $\sim 500 \mu \mathrm{m}$ thickness was used as a supporting layer, yielding $^{22}$

$$
\frac{P e}{d} \sim 0.465 \frac{c c}{\mathrm{~m}^{2} \cdot \mathrm{atm} \cdot \text { day }}=5.4 \times 10^{-17} \frac{\mathrm{m}}{\mathrm{Pa} \mathrm{s}}
$$

It is noted that the permeability of PUA film is higher than that of PET film by two orders of magnitude so that it can be neglected without loss of accuracy. ${ }^{23}$ In order to determine

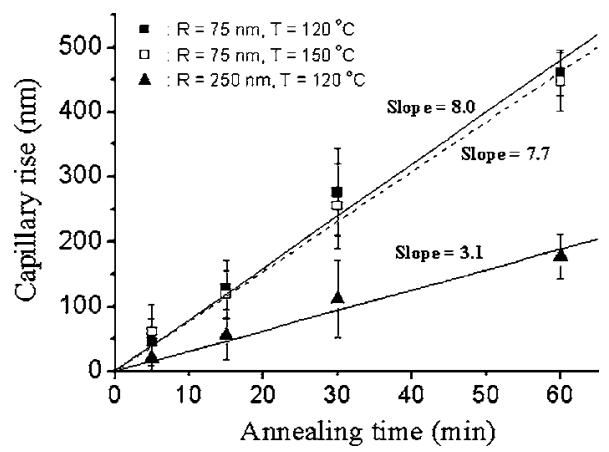

FIG. 3. Comparison between experimental data and theory for two different temperatures $\left(120,150{ }^{\circ} \mathrm{C}\right)$ and cavity diameters $(150,500 \mathrm{~nm}$ pillars). A linear increase was observed for all the conditions tested.

the contact angle of PMMA on PUA mold $(\theta)$, we used the geometric mean method, $\gamma_{l}(1+\cos \theta)=2\left(\gamma_{s}^{d} \gamma_{l}^{d}\right)^{1 / 2}$ $+2\left(\gamma_{s}^{p} \gamma_{l}^{p}\right)^{1 / 2}$ where the superscripts $d$ and $p$ are for the dispersion and polar components of the surface tension $\gamma^{24}$ Calculated dispersion and polar components surface tensions of PUA and PMMA were as follows: $\gamma_{\mathrm{PUA}}^{d}=21.6, \gamma_{\mathrm{PUA}}^{p}$ $=33.3(\mathrm{PUA}=$ solid $), \quad \gamma_{\mathrm{PMMA}}^{d}=39.89, \quad \gamma_{\mathrm{PMMA}}^{p}=3.17 \mathrm{~mJ} / \mathrm{m}^{2}$ (PMMA =liquid). From these values, $\theta=33.3^{\circ}$ was obtained. Using the same approach, we calculated the contact angle for polystyrene/PDMS system to be $67^{\circ}$, which is in excellent agreement with our previous report. ${ }^{3}$

Using Eqs. (1) and (2), the distance $z$ is calculated to be $\sim\left(3.75 \times 10^{-9} / R\right) t(R$ in $m, t$ in $s)$ or $(225 / R) t(R$ in $\mathrm{nm}, t$ in min). In Fig. 3, we compared the theoretical model with the experimental data with two different temperatures (120, $\left.150{ }^{\circ} \mathrm{C}\right)$ and cavity diameters $(150,500 \mathrm{~nm}$ pillars). A linear increase was observed for all the conditions tested, in good agreement with the theory. The best-fitting slope was $\sim 8$ for $150 \mathrm{~nm}$ pillars and $\sim 3$ for $500 \mathrm{~nm}$ pillars, respectively, which are slightly higher than the theoretically estimated values ( $\sim 3$ for $150 \mathrm{~nm}$ pillars and $\sim 0.9$ for $500 \mathrm{~nm}$ pillars). It is worthwhile noting that the filling rate was highly sensitive to the sealing conditions (or air trap) during the molding process. For example, the capillary rise for a portion of nanopillars on the same sample was several times faster than the theoretically estimated value, resulting in a complete filling into the cavity less than $10 \mathrm{~min}$ at $150{ }^{\circ} \mathrm{C}$. In this case, a significant variation in height was observed from edge to center due to nonuniform contact. To address this problem, we only considered the samples with good contact.

Interestingly, the slope was nearly the same for different temperatures above $T_{g}$ as shown in Fig. 3, which is another supporting evidence that the process is dominated by air permeation rather than viscosity (permeability decreases with increasing temperature by $273 / T$ where $T$ is in Kelvin). However, the capillary rise was retarded at late stage (i.e., slight parabolic profile), suggesting that the effects of viscosity and film thickness cannot be completely ruled out.

\section{Nanotribological tests using nanostructures with different tip shapes}

In the experiments, two types of nanostructures were observed depending on the fabrication temperature as shown in Fig. 4. When annealed at $120^{\circ} \mathrm{C}$ or less, most of the 
$\mathrm{T}=120^{\circ} \mathrm{C}$
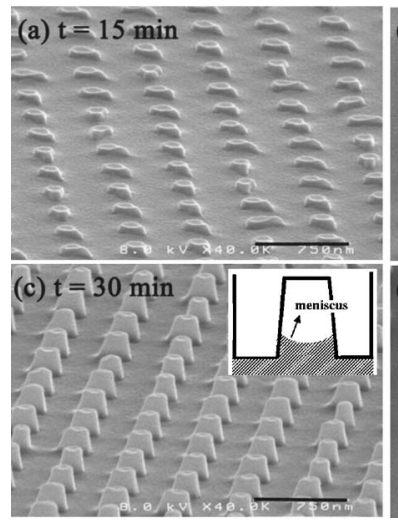

FIG. 4. Tilted SEM images for the two types of nanostructures at different temperatures: (a), (b) dimpled nanostructures at $120^{\circ} \mathrm{C}$ with time and (c), (d) spherical nanostructures at $150{ }^{\circ} \mathrm{C}$ with time. The scale bar indicates $750 \mathrm{~nm}$. Also shown in the inset are schematic illustrations for the formation of different curvature in the cavity.

nanostructures $(>\sim 80 \%)$ showed dimples at the tip [Figs. 2(a) and 2(c)], whereas the rest of the surface contained spheres or cones $(<\sim 20 \%)$. The height of dimples (defined as the height difference between the wall and the center) was found to decrease as the capillary rise progressed as determined from SEM images shown in Fig. 4. Apparently, the dimpled structured was formed by a meniscus and then maintained by a pressure concentration at the center of nanostructure. Further annealing led to the complete filling into the cavity as shown in Fig. 2.

When annealed at $150{ }^{\circ} \mathrm{C}$ or higher, on the other hand, most of the nanostructures $(>\sim 90 \%)$ were hemispherical in the initial stage [Fig. 2(b), $t=15 \mathrm{~min}$ ] and then evolved into a cone with a height of 330-390 nm [Fig. 2(d), $t=30 \mathrm{~min}$ ]. A small portion of dimpled or flat tips was found sporadically $(<\sim 10 \%)$. Also, further annealing yielded the complete filling into the cavity (not shown). Interestingly, the meniscus was not found in this case, rendering spheres or cones with an inverted curvature at all times presumably due to reflows of polymer melt. In a separate experiment in which the samples were annealed at $135^{\circ} \mathrm{C}$, the surface contained a mixture of cones and dimples with nearly equal populations (see Fig. 5), suggesting that temperature is a crucial factor in determining the shape of nanostructure. In addition to the

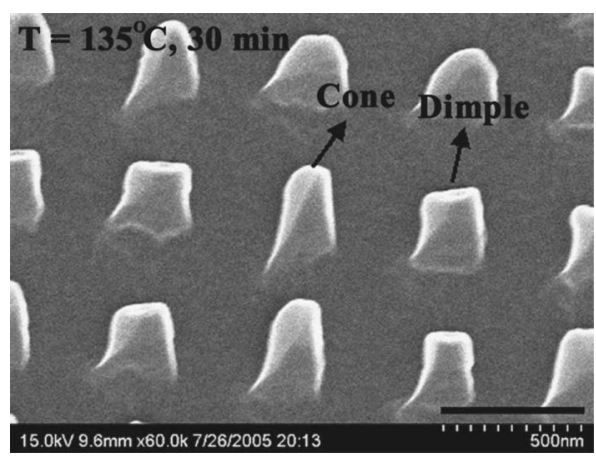

FIG. 5. A SEM image for the combination of cones and dimples when annealed at $135{ }^{\circ} \mathrm{C}$ for $30 \mathrm{~min}$. The scale bar indicates $500 \mathrm{~nm}$.

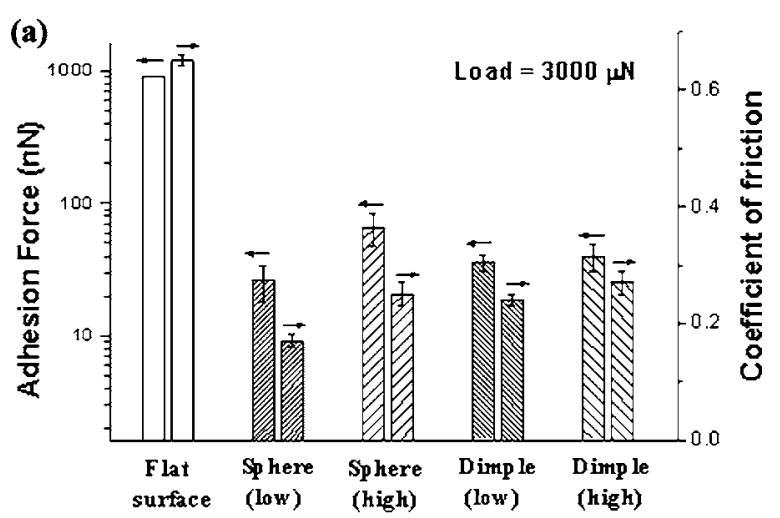

(b)

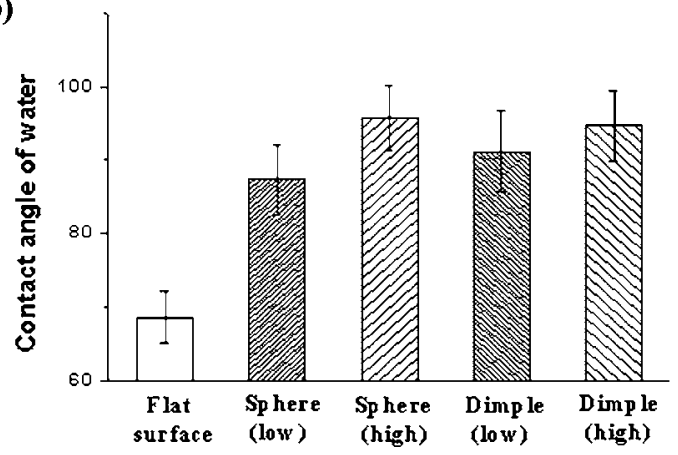

FIG. 6. (a) Comparison of adhesion force at nano-scale and coefficient of friction at microscale for different tip shapes and heights. (b) Measurements of contact angle of water on various nanostructured surfaces. Errors are within $5 \%$.

temperature, modification of mold geometry or wetting conditions would result in a different shape of nanostructure, for which a further study would be required.

From a tribological point of view, the fabrication of nanostructures with different tip shape would provide an insight into potential nanotribological applications. ${ }^{25,26} \mathrm{Re}-$ cently, we reported that nanopatterns with three different aspect ratios made of PMMA showed superior tribological properties at both micro- and nanoscale. ${ }^{27}$ As a separate experiment to elucidate the effects of tip shape of nanostructure, we measured adhesion force and coefficient of friction using AFM and a microtribo tester, respectively. ${ }^{28}$ In order to minimize errors in measurements, the nanopillar patterns were generated over a large area up to $\sim 5 \times 5 \mathrm{~cm}^{2}$ and then the patterned substrate was cut into small pieces of 1 $\times 1 \mathrm{~cm}^{2}$ prior to tribological tests.

As shown in Fig. 6(a), the adhesion force was in general significantly reduced on a nanostructured surface compared to a flat PMMA film regardless of the shape of nanostructure (sphere or dimple), which agrees well with our previous report. ${ }^{28}$ Of the nanostructures tested, the hemispheres denoted as "sphere (low)" [also see Fig. 4(b)] provided the maximum reduction in adhesion force, whereas the cones denoted as "sphere (high)" [Fig. 4(d)] showed the minimum reduction. The other two dimpled nanopatterns showed almost the same adhesion forces, suggesting that the dimpled shape is more important than the height.

The origin of the reduced adhesion is ascribed to two contributions: one is the reduced contact area and the other is the increased hydrophobicity. Measurements of contact angle 
of water shown in Fig. 6(b) indicated that the nanostructured surface was more hydrophobic than the flat surface because of air pocket in the spaces between nanostructures and roughness effects as can be seen in the so-called "Lotus effect." ${ }^{29}$ Apparently, the contact angles are independent of the tip shape, since the solid fraction in contact with a water droplet is nearly the same for a given mold geometry. Based on these observations, it is unlikely that the capillary force dominates the adhesion force. Also, the adhesion force increased with increasing height of nanostructure (aspect ratio) for a given mold geometry, which is due to the increase in the projected area.

The friction resistance of the nanopatterns at microscale also exhibited a large reduction for all the structures tested when compared to a flat surface as shown in Fig. 6(a). This is attributed to the reduced contact area and lower inherent adhesion. Furthermore, in the case of high aspect ratio structures, partial bending (elastic deformation) of the pillars may take place, which would in turn increase the contact area and then the friction resistance. ${ }^{27}$ A notable finding here is that the spherical shape is superior to the dimpled one in reducing the coefficient of friction. This is readily understood in that the partial sliding of the measuring tip occurs on top of the spherical surface, leading to enhanced lubrication properties. This would be particularly important in tailoring the tip of nanostructures for a specific application without resorting to other multi-step, complex methods.

\section{SUMMARY}

We have presented a simple capillary kinetic model to describe the capillary rise of polymer melt into a less permeable mold. The kinetic model predicts that the capillary rise is linearly proportional to time for less permeable molds, which has been verified using PUA molds with PET film as a supporting layer. A similar situation can be found in high temperature nanoimprint lithography when a negative mold is used. ${ }^{30}$ It has been shown that, for a given mold geometry, the height of nanostructure is tunable by changing the annealing time. Also the tip shape can be rounded or dimpled depending on the temperature as a result of reflows of polymer melt. For nanotribological applications, the effects of tip shape of nanostructure have been tested. It was found that both adhesion and friction forces were significantly reduced on the nanostructured surface due to the reduced contact area and lubrication effects at the tip. It is envisioned that this simple approach provides a convenient route to shapeengineering surface nanotopography for better nanotribological properties.

\section{ACKNOWLEDGMENTS}

This work was supported by the Micro Thermal System Research Center of Seoul National University. K.Y.S. thanks Professor H. H. Lee for providing the surface tension values of PUA mold and PMMA.

${ }^{1}$ A. W. Adamson and A. P. Gast, Physical Chemistry of Surfaces, 6th ed. (Wiley, New York, 1997).

${ }^{2}$ E. Kim, Y. N. Xia, and G. M. Whitesides, Nature (London) 376, 581 (1995).

${ }^{3}$ K. Y. Suh, Y. S. Kim, and H. H. Lee, Adv. Mater. (Weinheim, Ger.) 13, 1386 (2001).

${ }^{4}$ Y. S. Kim, K. Y. Suh, and H. H. Lee, Appl. Phys. Lett. 79, 2285 (2001).

${ }^{5}$ K. Y. Suh and H. H. Lee, Adv. Funct. Mater. 12, 405 (2002).

${ }^{6}$ H. E. Jeong and K. Y. Suh, J. Appl. Phys. 97, 114701 (2005).

${ }^{7}$ E. Delamarche, H. Schmid, B. Michel, and H. Biebuyck, Adv. Mater. (Weinheim, Ger.) 9, 741 (1997).

${ }^{8}$ A. Bietsch and B. Michel, J. Appl. Phys. 88, 4310 (2000).

${ }^{9}$ C. Y. Hui, A. Jagota, Y. Y. Lin, and E. J. Kramer, Langmuir 18, 1394 (2002).

${ }^{10} \mathrm{H}$. Schmid and B. Michel, Macromolecules 33, 3042 (2000).

${ }^{11}$ T. W. Odom, J. C. Love, D. B. Wolfe, K. E. Paul, and G. M. Whitesides, Langmuir 18, 5314 (2002).

${ }^{12}$ K. M. Choi and J. A. Rogers, J. Am. Chem. Soc. 125, 4060 (2003).

${ }^{13}$ G. Csucs, T. Kunzler, K. Feldman, F. Robin, and N. D. Spencer, Langmuir 19, 6104 (2003)

${ }^{14}$ K. Y. Suh, R. Langer, and J. Lahann, Appl. Phys. Lett. 83, 4250 (2003).

${ }^{15}$ D. Y. Khang and H. H. Lee, Adv. Mater. (Weinheim, Ger.) 16, 176 (2004).

${ }^{16}$ D. Y. Khang, H. Kang, T. Kim, and H. H. Lee, Nano Lett. 4, 633 (2004).

${ }^{17}$ Y. S. Kim, H. H. Lee, and P. T. Hammond, Nanotechnology 14, 1140 (2003).

${ }^{18}$ S. J. Choi, P. J. Yoo, S. J. Baek, T. W. Kim, and H. H. Lee, J. Am. Chem. Soc. 126, 7744 (2004)

${ }^{19}$ P. J. Yoo, S. J. Choi, J. H. Kim, D. Suh, S. J. Baek, T. W. Kim, and H. H. Lee, Chem. Mater. 16, 5000 (2004).

${ }^{20}$ T. C. Merkel, V. I. Bondar, K. Nagai, B. D. Freeman, and I. Pinnau, J. Polym. Sci., Part B: Polym. Phys. 38, 415 (2000).

${ }^{21}$ K. Y. Suh, P. Kim, and H. H. Lee, Appl. Phys. Lett. 85, 4019 (2004).

${ }^{22}$ N. Qureshi, E. V. Stepanov, D. Schiraldi, A. Hiltner, and E. Baer, J. Polym. Sci., Part B: Polym. Phys. 38, 1679 (2000).

${ }^{23}$ H. B. Park and Y. M. Lee, J. Membr. Sci. 197, 283 (2002).

${ }^{24}$ S. M. Seo, J. Y. Park, and H. H. Lee, Appl. Phys. Lett. 86, 133114 (2005).

${ }^{25}$ B. Bhushan, Wear 229, 465 (1999).

${ }^{26}$ Z. Burton and B. Bhushan, Nano Lett. 5, 1607 (2005).

${ }^{27}$ E. S. Yoon, R. A. Singh, H. S. Kong, B. Kim, D. H. Kim, H. E. Jeong, and K. Y. Suh, Tribol. Lett. 21, 36 (2006).

${ }^{28}$ E. S. Yoon, S. H. Yang, H. Kong, and K. H. Kim, Tribol. Lett. 15, 145 (2003).

${ }^{29}$ C. Neinhuis and W. Barthlott, Ann. Bot. (London) 79, 667 (1997).

${ }^{30}$ H. C. Scheer, H. Schulz, T. Hoffmann, and C. M. S. Torres, J. Vac. Sci. Technol. B 16, 3917 (1998) 